\title{
ASPECTOS COMPARATIVOS DA EVOLUÇÃO ACADÊMICA DE ALUNOS DO CURSO DE LICENCIATURA EM CIÊNCIAS BIOLÓGICAS DE REGIÕES DISTINTAS DO ESTADO DO RIO DE JANEIRO
}

\author{
Celly Cristina Alves do Nascimento Saba | celly@uerj.br \\ Doutora em Ciências pela Universidade Federal do Rio de Janeiro - UFRJ. Professora Adjunta da Universidade do Estado do Rio de \\ Janeiro - UERJ e Coordenadora do Curso de Licenciatura em Ciências Biológicas UERJ/CEDERJ
}

Carmem Adilia Simões da Fonseca | carmemad@ig.com.br

Doutora em Ciências pela Universidade Federal do Rio de Janeiro - UFRJ. Tutora a Distância da Disciplina Corpo Humano II - Curso de Licenciatura em Ciências Biológicas - EAD. Professora Adjunta da Universidade Estácio de Sá.

Daniela Beltrão de Souza | danielab@uerj.br

Graduada em Pedagogia pela Universidade do Estado do Rio de Janeiro - UERJ. Assistente da Coordenação do Curso de Licenciatura em Ciências Biológicas UERJ/CEDERJ

Marisa Maria Dreyer Breitenbach | marisadreyer@globo.com Doutora em Ciências pela Universidade Federal do Rio de Janeiro - UFRJ. Professora Adjunta da Universidade do Estado do Rio de Janeiro - UERJ e Coordenadora da Disciplina Corpo Humano II.

\section{Resumo}

Este estudo visa analisar o desempenho dos prováveis formandos 2009.2 do curso de Licenciatura em Ciências Biológicas, oferecido na modalidade semipresencial pelo Consórcio CEDERJ, através da ponderação de dados acadêmicos de 13 estudantes matriculados no polo Bom Jesus do Itabapoana (BJE) e 15 matriculados no polo Paracambi (PAR). A análise comparativa baseou-se nos resultados alcançados no primeiro semestre, no desempenho na disciplina Corpo Humano II - $\mathrm{CH} 2$ ( $9^{\circ}$ período da grade curricular), no número de períodos para integralização do curso e no coeficiente de rendimento alcançado até 2009.1. As apreciações iniciais demonstraram a obtenção de médias mais altas no polo BJE. Tal fato é corroborado pela assiduidade na utilização das ferramentas de apoio - Plataforma CEDERJ e sistema telefônico 0800 - e pela formação de grupos de estudo no polo BJE. A análise do coeficiente de rendimento atingido em 2009.1 evidenciou sua relação direta com as médias de $\mathrm{CH} 2$, e de ambos com o número de períodos recomendados à integralização do curso. Novamente, o maior aproveitamento se deu com os matriculados no polo BJE. Outro parâmetro destaca maior percentual de formandos no polo BJE, apesar do menor número de inscritos. A partir desse diagnóstico inicial, buscamos conhecer a evolução acadêmica dos alunos do Curso de Licenciatura em Ciências Biológicas que atuarão como docentes no interior do estado do Rio de Janeiro.

\section{Palavras-chave}

Educação a Distância. Formação docente. Ensino de Ciências e Biologia. 
Aspectos comparativos da evolução acadêmica de alunos do curso de licenciatura em Ciências Biológicas de regiōes distintas do Estado do Rio de Janeiro _

A COMPARATIVE ANALYSIS OF ACADEMIC ACHIEVEMENT OF A BIOLOGICAL SCIENCES TEACHER TRAINING COURSE STUDENTS IN TWO DIFFERENT REGIONS OF RIO DE JANEIRO STATE

\begin{abstract}
This study aims to analyze the achievement of students (2009.2) concluding a CEDERJ Consortium teacher training course in Biological Sciences carried out in semi-distance modality, through analysis of academic data of 13 students enrolled at Bom Jesus de Itabapoana (BJE) and of 15 enrolled at Paracambi (PAR). The comparative analysis was based on student results during the first class semester (2009.1) in the subject Human Body II - CH2 (9th grade in the course curriculum), the number of semesters needed for course completion and the cumulative grade average up to 2009.1. Initial analysis revealed higher grade averages at the BJE center. This result is corroborated by students' assiduous use of supporting tools (CEDERJ virtual support and 0800 call center) and by the formation of study groups at the BJE center. There was a direct correlation between student grade averages in 2009.1 and the $\mathrm{CH} 2$ averages, as well as between both and the number of semesters recommended for completing the course. Once again, BJE students showed a better performance. Also, even though there were fewer students enrolled at BJE, more students completed the course. From this initial diagnosis, we aim to follow the academic progress of the students enrolled in the Biological Sciences teacher training course who in the future will teach in schools around Rio de Janeiro State.
\end{abstract}

\section{Keywords}

Distance education. Teacher training. Science and Biology teaching.

\section{Introdução}

Um dos pontos mais prementes na atualidade, no que tange à educação superior, vincula-se à sua democratização e à ampliação ao seu acesso. Buscando fomentar e cooperar para tornar real essa questão e, do mesmo modo, abarcar a população de diferentes regiões do estado do Rio de Janeiro, o Governo do estado do Rio de Janeiro e as universidades públicas do Estado celebraram o Consórcio CEDERJ. Dentre os cursos desenvolvidos no âmbito do Consórcio temos o curso de Licenciatura em Ciências Biológicas, cujo objetivo prima pela formação de docentes de Ciências e Biologia.

Sua coordenação é compartilhada pelas universidades consorciadas: Universidade do Estado do Rio Janeiro (UERJ), Universidade Estadual do Norte Fluminense (UENF) e Universidade Federal do Rio de Janeiro (UFRJ). Sob coordenação da UERJ estão os polos de Nova Friburgo, Paracambi e Resende, e, nesta universidade, o curso tem suas bases estabelecidas por meio da coordenação do curso, da coordenação de tutoria e da coordenação de sete disciplinas.

Aglutinando tal estrutura a uma metodologia peculiar, o curso de Licenciatura em Ciências Biológicas a Distância apresenta grade curricular de 10 semestres, em disciplinas que totalizam 3.720 horas, estando organizado para que a aprendizagem seja alcançada em caráter semipresencial.

Diante desse panorama, o presente estudo visa analisar o desempenho dos prováveis formandos do referido curso no semestre 2009.2. Foram ponderados dados acadêmicos de estudantes matriculados em dois diferentes polos, oportunizando, dessa forma, uma reflexão acerca da real contribuição do curso no estado do Rio de Janeiro.

\section{Detalhamento do desenvolvimento acadêmico no curso de Licenciatura em Ciências Biológicas}

É sabido que a distância dos grandes centros metropolitanos pode afetar a formação básica dos indivíduos e que no interior se somam outras dificuldades que podem limitar seu desenvolvimento. No entanto, em relação ao conhecimento, carac- 
terísticas espaço-temporais foram parcialmente superadas na medida em que o desenvolvimento de Tecnologias de Comunicação e informação (TICs) consistentes permitiram que a "informação viajasse de forma independente de seus portadores físicos $e$ independente também dos objetos sobre os quais informava" (BAUMAN, 1999, p. 21.) Dessa forma, a separação dos movimentos da informação em relação aos movimentos de seus portadores ocasiona impactos na formação dos discentes, bem como no processo ensino-aprendizagem.

No Rio de Janeiro, a busca pela ampliação do acesso ao Ensino Superior gratuito e de qualidade e ainda a necessidade de interiorização atuaram como mola propulsora da formação do Consórcio CEDERJ.

O CEDERJ foi instituído a partir da celebração de um consórcio entre a Secretaria de Ciência e Tecnologia (SECT) e as universidades públicas do estado do Rio de Janeiro, em janeiro de 2000. Foi idealizado a partir da necessidade de formar e manter profissionais qualificados em seus municípios de origem, dada a carência de profissionais da área de educação para o Ensino Fundamental e Médio da rede pública, sendo a melhor participação social no desenvolvimento das regiões do estado um dos objetivos do Consórcio.

Em relação ao curso de Ciências Biológicas, as universidades responsáveis pela construção e pela coordenação são UERJ, UENF e UFRJ. No momento, o curso vem sendo oferecido em 17 polos regionais dentre os 33 do Consórcio CEDERJ, possuidores de infraestrutura capaz de propiciar o desenvolvimento do aluno, bem como relacioná-lo à universidade que irá diplomá-lo.

A estrutura curricular do curso foi construída agregando disciplinas e docentes de todas as instituições envolvidas, que compartilham seus saberes e experiências. Cabe ressaltar que o curso foi organizado seguindo os mesmos moldes dos cursos presenciais oferecidos pelas universidades consorciadas, mantendo a reconhecida qualidade de ensino das referidas instituições.

Para promover o aprendizado contemplando a autonomia do aluno, o CEDERJ oferece recursos de mediação, apropriação de conteúdos, desen- volvimento de habilidades e competências, através do ambiente virtual de aprendizagem - Plataforma CEDERJ, de linha telefônica em sistema gratuito 0800 -, de material didático impresso e de atividades tutoriais.

A estrutura da Plataforma CEDERJ possibilita a elaboração de fóruns temáticos, chats, a socialização de informações, a discussão específica de conteúdo pela sala de tutoria, avaliações e gabaritos, a disponibilização do material didático etc. Completando o ciclo de interação discente-disciplina, o sistema semipresencial conta com o auxílio de tutores presenciais, que, atuando nos polos, orientam atividades de aulas práticas realizadas nos laboratórios, estudos dirigidos, atividades de campo e atividades desenvolvidas em escolas; e tutores a distância, que ficam nas universidades em contato direto com os coordenadores de disciplinas e prestam atendimento diário aos discentes pela Plataforma, e em sistema de plantão pelo atendimento telefônico.

Objetivando manter a sinergia entre o planejamento e a metodologia aplicados no processo ensino-aprendizagem, constantes avaliações são efetuadas e, quando necessário, tais aspectos passam por adequações.

Nessa perspectiva, este estudo teve como objetivo avaliar o desempenho de alunos prováveis formandos de 2009.2, do curso de Licenciatura em Ciências Biológicas na modalidade semipresencial do Consórcio CEDERJ, oriundos de polos cujas características regionais são bastante distintas.

A análise comparativa foi efetuada com base nos resultados alcançados pelos alunos em seu primeiro semestre de curso, o desempenho em uma disciplina do $9^{\circ}$ período da grade curricular, o número de períodos para integralização do curso e o coeficiente de rendimento alcançado até 2009.1.

Para tanto, dentre os 17 polos dois foram escolhidos Um dos polos ao norte. mais distante da capital, localizado no município de Bom Jesus do Itabapoana (BJE), e um dos polos ao sul, no município de Paracambi (PAR), próximo às universidades e localizado em um centro de tecnologia. Os prováveis formandos do polo ao sul totalizam 15 alunos, enquanto os do polo ao norte totalizam em 13 , com matrícula em diferentes semestres (Figuras 1A e 1B). 
Aspectos comparativos da evolução acadêmica de alunos do curso de licenciatura em Ciências Biológicas de regiōes distintas do Estado do Rio de Janeiro _

Figura 1: Identificação dos prováveis formandos de 2009.2.

Polo Paracambi (PAR)

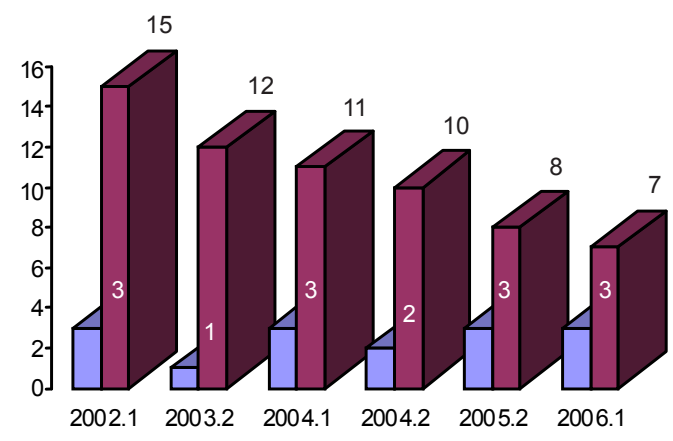

Início do Curso

$\mathrm{n}^{\circ}$ de alunos
Polo Bom Jesus do Itabapoana (BJE)

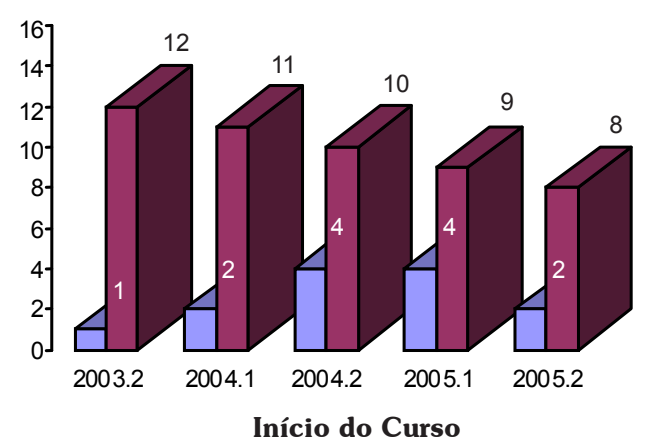

períodos letivos $\mathrm{p} /$ conclusão do curso

Fonte: Gráficos gerados a partir de dados obtidos no sistema acadêmico CEDERJ

O desempenho nas disciplinas de $1^{\circ}$ período, Grandes Temas em Biologia (GTB), Diversidade dos Seres Vivos (DSV), Introdução à Informática (IINF), Dinâmica da Terra (DT) e Bioquímica I (BQ1) foi comparado ao desempenho na disciplina de $9^{\circ}$ período, Corpo Humano II (CH2). A disciplina Corpo Humano II foi inserida como parâmetro de comparação, tendo em vista ser considerada, pelos discentes, como complexa, por abordar o funcionamento do corpo humano de forma integrada, contextualizando-o com questões de saúde pública mais incidentes na sociedade brasileira. Verificamos que o desempenho no polo Bom Jesus do Itabapoana (BJE) foi superior ao do polo Paracambi (PAR). As notas médias alcançadas em BJE no $1^{\circ}$ período variaram entre 6,3 e 9,1, em Grandes Temas em Biologia (GTB); entre 2,3 a 9,5 em Diversidade dos Seres Vivos (DSV); entre 5,3 e 8,8 em Introdução à Informática (IINF); entre 4,1 e 8,4 em Dinâmica da Terra (DT); entre 3,5 e 8,7 em Bioquímica I (BQ1) e entre 2,6 e 9,4 em CH2. Já em PAR as notas alcançadas variaram em GTB entre 1,0 e 9,5; em DSV entre 5,1 e 9,5; em IINF entre 6,2 e 9,0; em DT entre 4,7 e 8,8; em BQ1 entre 0,5 e 5,5 e em $\mathrm{CH} 2$ variaram entre 6,0 e 8,6 (Figuras 2 e $\mathbf{3}$ ). As notas indicadas se referem à primeira inscrição do aluno na disciplina, não sendo levada em conta a nota obtida quando o mesmo faz a disciplina mais de uma vez para alcançar a aprovação.

Figura 2: Notas médias nas disciplinas de $1^{\circ}$ período
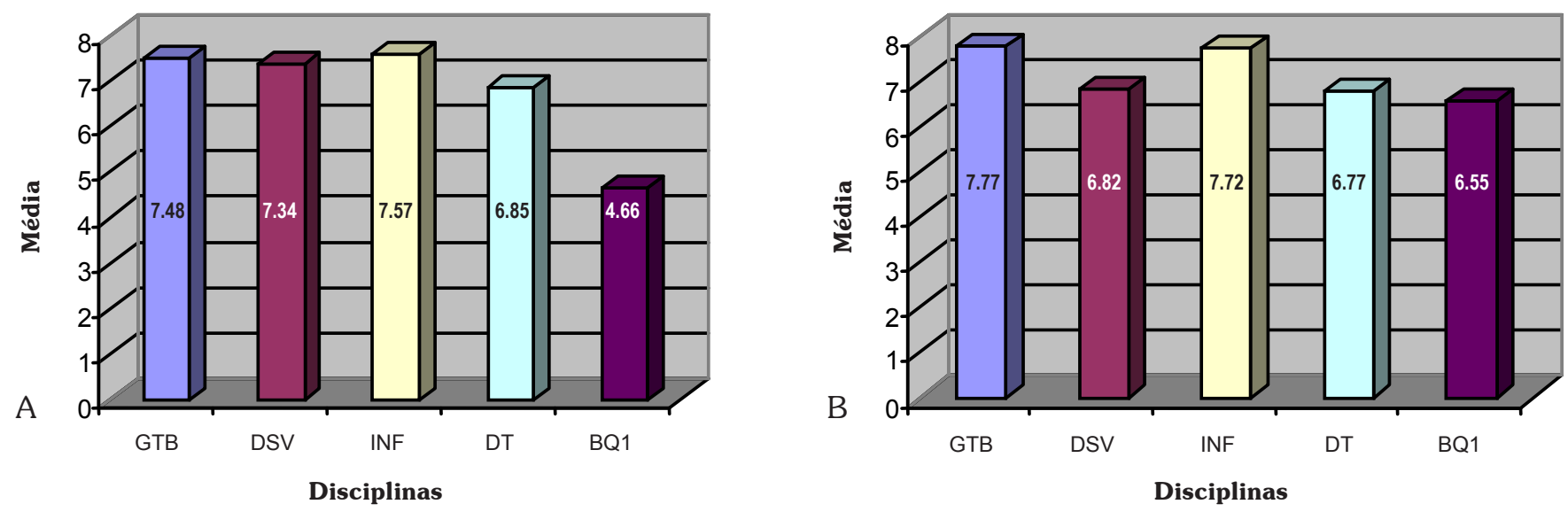

Fonte: Gráficos gerados a partir de dados obtidos no sistema acadêmico CEDERJ. 
Figura 3: Médias gerais das disciplinas de $1^{\circ}$ período

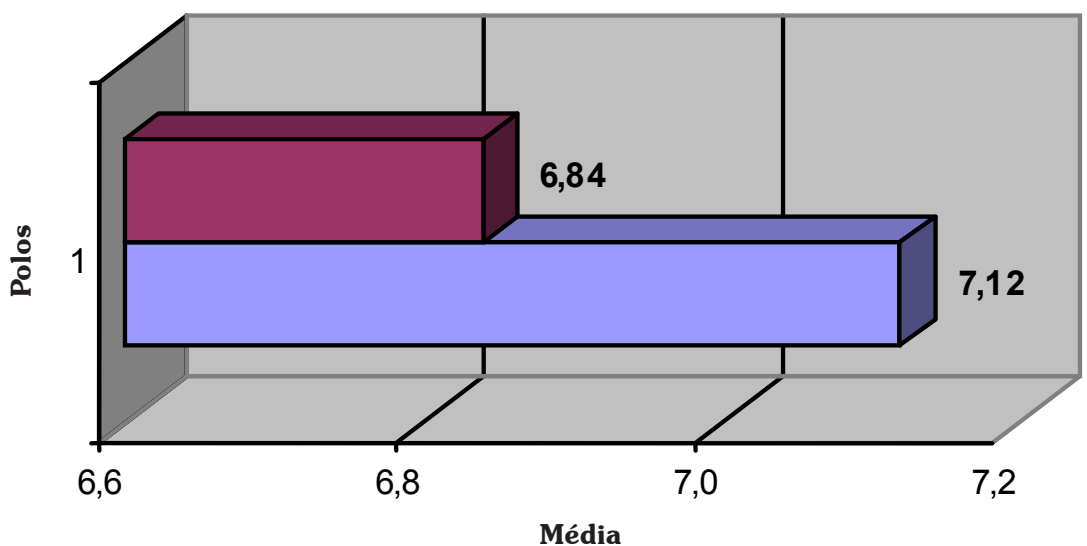

Polo Paracambi (PAR)

Polo Bom Jesus do Itabapoana (BJE)

Fonte: Gráficos gerados a partir de dados obtidos no sistema acadêmico CEDERJ

Um fato de relevância para a diferença nos resulta- apoio, a Plataforma e o 0800, bem como a formação dos obtidos pelos alunos de BJE na disciplina $\mathrm{CH} 2$ de grupos de estudo no polo, conforme relato da tufoi a utilização mais frequente das ferramentas de tora a distância da disciplina (Quadro 1).

Quadro 1: Notas médias dos prováveis formandos 2009.2 na disciplina CH2.

\begin{tabular}{|c|c|c|c|c|c|c|c|c|c|c|c|c|c|c|c|c|}
\hline Alunos / Polos & 1 & 2 & 3 & 4 & 5 & 6 & 7 & 8 & 9 & 10 & 11 & 12 & 13 & 14 & 15 & Média \\
\hline PAR & 7,4 & 8,6 & 0,8 & 6,2 & 7,2 & 0,5 & 6,6 & 6,5 & 0,9 & 7,9 & 7,2 & 7,2 & 8,0 & 7,7 & 6,1 & 5,92 \\
\hline BJE & 6,3 & 9,2 & 2,5 & 6,0 & 7,6 & 6,4 & 6,0 & 7,9 & 4,4 & 9,4 & 2,6 & 4,7 & 5,8 & - & - & 6,10 \\
\hline
\end{tabular}

Fonte: Gráficos gerados a partir de dados obtidos no sistema acadêmico CEDERJ.

Quando o desempenho foi avaliado pelo coeficiente de rendimento atingido em 2009.1, verificamos que esse parâmetro apresenta uma relação direta com as médias de CH2 (Figura 4), e que ambos estão relacionados ao número de períodos prováveis para a integralização do curso.
Assim, a ideia inicial de maior aproveitamento permanece para os matriculados no polo BJE, os quais apresentaram coeficiente de rendimento variando entre 5,3 e 8,1, enquanto os matriculados em PAR apresentaram variação do coeficiente de rendimentos entre 3,2 e 7,7.

Figura 4: Coeficiente de rendimento (CR) e média em $\mathrm{CH} 2$ dos prováveis formandos de 2009.2

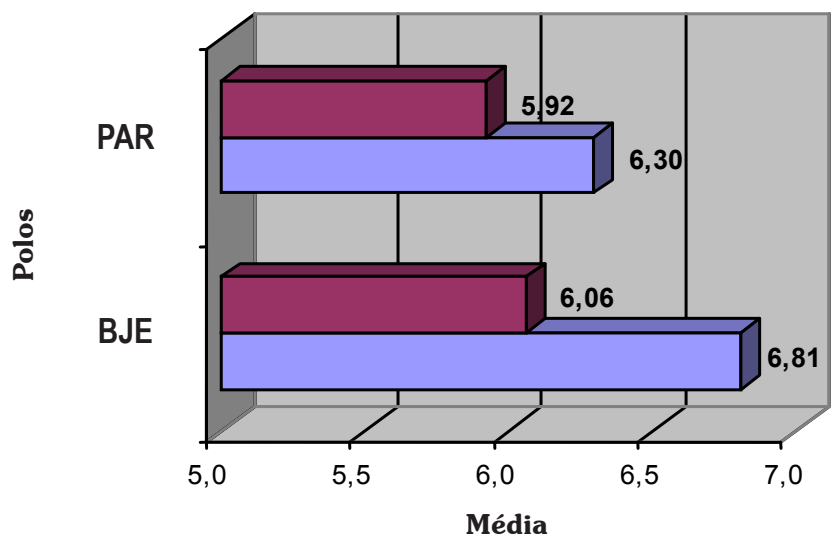

Média $\mathrm{CH} 2$

Média CR

Fonte: Gráficos gerados a partir de dados obtidos no sistema acadêmico CEDERJ. 
Aspectos comparativos da evolução acadêmica de alunos do curso de licenciatura em Ciências Biológicas de regiôes distintas do Estado do Rio de Janeiro _ EAD

Apesar do tempo de integralização ser quase 0,5 período para o conjunto de alunos analisados o mesmo, em média no norte há um ganho de (Figura 5).

Figura 5: Tempo médio de integralização dos alunos de PAR e BJE.

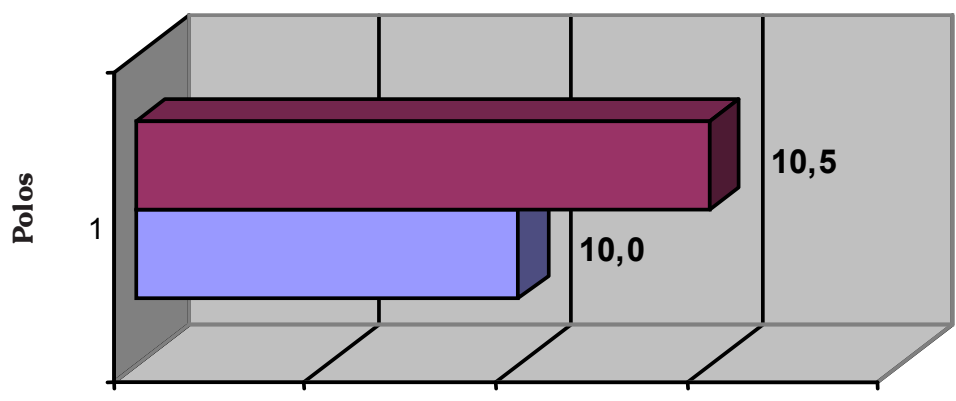

Média de períodos para conclusão do Curso
Paracambi

Bom Jesus do Itabapoana

Fonte: Gráficos gerados a partir de dados obtidos no sistema acadêmico CEDERJ

Em números gerais, em ambos os polos, elementos como trancamento de matrícula e cancelamento foram majoritários. Evidenciamos ainda que, ape- sar do menor número de inscritos no polo ao norte, BJE, o percentual de formandos foi maior, ou seja, $5 \%$, comparados aos 3\% de PAR (Figura 6).

Figura 6: Número total de alunos nos polos.

\section{A - Paracambi $\quad$ B - Bom Jesus do Itabapoana}
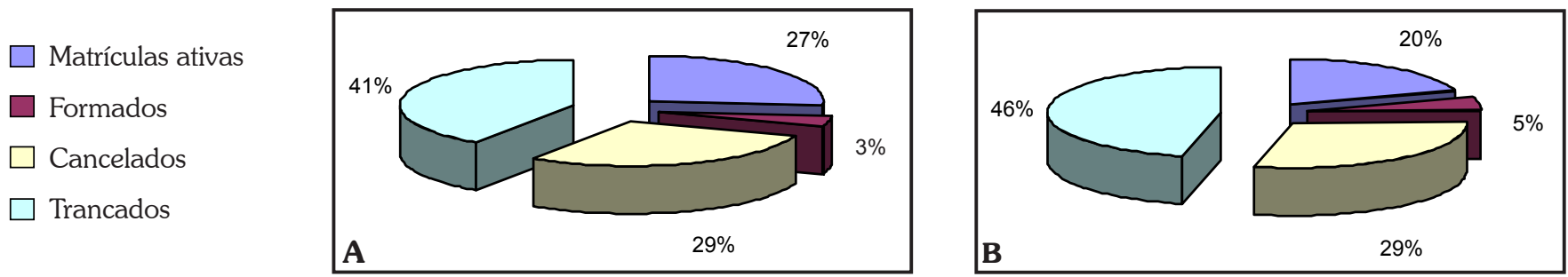

Fonte: Gráficos gerados a partir de dados obtidos no sistema acadêmico CEDERJ

\section{Considerações finais}

De acordo com Lévy (1999), o estilo pedagógico proposto pela Educação a Distância - EAD - favorece, simultaneamente, as aprendizagens personalizadas e a aprendizagem coletiva em rede ao vincular as hipermídias, as redes de comunicação interativas $e$ as tecnologias intelectuais da cibercultura às interações próprias do processo ensino-aprendizagem. A essência desse estilo revela o caráter libertador da EAD (FREIRE, 2000), tendo em vista que tanto os docentes quanto os discentes são sujeitos cognitivos $e$ agentes críticos do ato de conhecer quando desenvolvem em comunhão a aprendizagem personalizada $e$ a aprendizagem coletiva em rede.

Através deste pequeno estudo buscamos conhecer um pouco melhor a evolução acadêmica dos alunos do curso de Licenciatura em Ciências Biológicas par- tindo do viés libertador do qual a proposta pedagógica do Consórcio CEDERJ está imbuída.

Consideramos que o bom desempenho no $1^{\circ}$ período contribui para o incentivo à manutenção do ritmo de estudo, diminuindo as reprovações $e$ assim tornando o tempo de integralização, sugerido em 10 períodos, viável. Além disso, demonstra que as dificuldades vão diminuindo, tornando o aluno mais adaptado à modalidade semipresencial, capaz de obter bom desempenho em disciplinas consideradas (pelos discentes) como de grande complexidade/dificuldade, como Corpo Humano II (CH2). A maturidade educacional sendo alcançada torna o discente consciente de suas necessidades e de como buscar a solução, tirando maior proveito dos recursos oferecidos para a construção do conhecimento. Dentre as várias opções abertas ao discente, alguns 
aspectos relatados por componentes dos grupos estudados constatam que a formação de grupos/turmas menores facilita a organização. Apesar da grande distância da capital, em geral, a utilização dos instrumentos de apoio oferecidos para a construção do conhecimento, como o 0800 e a Plataforma, é maior. Em polos mais próximos à região metropolitana, a maior facilidade de acesso contribui para a formação de turmas heterogêneas, permitindo que indivíduos de vários municípios vizinhos, inclusive da capital se matriculem no polo. No entanto, esse ponto parece contribuir para a dispersão e não para o agrupamento dos discentes, o que pode ser um fator de influência negativa sobre a construção do aprendizado e consequentemente, do resultado es- perado. Pode ainda ser desestimulante em face do deslocamento necessário para algumas atividades e, portanto, levar à baixa procura pelos instrumentos de apoio. Dessa maneira, diminui o rendimento $e$ aumenta o tempo de integralização do curso, quando não leva ao abandono.

Logo, fica claro que o objetivo do Consórcio CEDERJ de contribuir para a formação de professores de Ciências e Biologia para o Ensino Fundamental e Médio, no interior do estado do Rio de Janeiro, visando suprir a demanda da rede pública, está sendo concretizado. Sendo uma modalidade recente de ensino, apresenta resultados significativos que tendem a se fortalecer, com o reconhecimento de sua qualidade. 
Aspectos comparativos da evolução acadêmica de alunos do curso de licenciatura em Ciências Biológicas de regiôes distintas do Estado do Rio de Janeiro _

\section{Referências bibliográficas:}

BAUMAN, Zygmunt. Globalização: as conseqüências humanas. Tradução de Marcus Penchel. Rio de Janeiro: Jorge Zahar, 1999.

FREIRE, Paulo; SHOR, Ira. Medo e ousadia: cotidiano do professor. 8. ed. Rio de Janeiro: Paz e Terra, 2000.

LEVY, Pierre. Cibercultura. Rio de Janeiro: Editora 34, 1999. 